City University of New York (CUNY) CUNY Academic Works

\title{
Automated Refactoring of Legacy Java Software to Default Methods
}

Raffi T. Khatchadourian

CUNY Hunter College

Hidehiko Masuhara

Tokyo Institute of Technology

\section{How does access to this work benefit you? Let us know!}

More information about this work at: https://academicworks.cuny.edu/hc_pubs/287

Discover additional works at: https://academicworks.cuny.edu

This work is made publicly available by the City University of New York (CUNY).

Contact: AcademicWorks@cuny.edu 


\section{Automated Refactoring of Legacy Java Software to Default Methods}

\author{
Raffi Khatchadourian \\ City University of New York \\ raffi.khatchadourian@hunter.cuny.edu
}

\author{
Hidehiko Masuhara \\ Tokyo Institute of Technology \\ masuhara@acm.org
}

\begin{abstract}
Java 8 default methods, which allow interfaces to contain (instance) method implementations, are useful for the skeletal implementation software design pattern. However, it is not easy to transform existing software to exploit default methods as it requires analyzing complex type hierarchies, resolving multiple implementation inheritance issues, reconciling differences between class and interface methods, and analyzing tie-breakers (dispatch precedence) with overriding class methods to preserve type-correctness and confirm semantics preservation. In this paper, we present an efficient, fully-automated, type constraint-based refactoring approach that assists developers in taking advantage of enhanced interfaces for their legacy Java software. The approach features an extensive rule set that covers various corner-cases where default methods cannot be used. To demonstrate applicability, we implemented our approach as an Eclipse plug-in and applied it to 19 real-world Java projects, as well as submitted pull requests to popular GitHub repositories. The indication is that it is useful in migrating skeletal implementation methods to interfaces as default methods, sheds light onto the pattern's usage, and provides insight to language designers on how this new construct applies to existing software.
\end{abstract}

Keywords-refactoring; java; interfaces; default methods

\section{INTRODUCTION}

With the introduction of enhanced interfaces in Java 8, developers can now write default (instance) methods, which include an implementation that implementers will inherit if they do not provide their own [1]. Although the original motivation was to enable developers to add new functionality to existing interfaces without breaking clients [2], default methods can be used [3] as a replacement of the skeletal implementation pattern [4. Item 18]. This pattern centers around creating an abstract skeletal implementation class, which implementers extend, that provides a partial interface implementation, making the interface easier to implement.

While there are many advantages in migrating legacy code using the skeletal implementation pattern to instead use default methods, e.g., foregoing the need for subclassing, having classes inherit behavior (but not state) from multiple interfaces [3], facilitating local reasoning [5], doing so may require significant manual effort, especially in large projects. Particularly, there are subtle language and semantic restrictions, e.g., interfaces cannot declare instance fields. It requires preserving type-correctness by analyzing complex type hierarchies, resolving issues arising from multiple (implementation) inheritance, reconciling differences between class and interface methods, and ensuring tie-breakers with overriding class methods, i.e., rules governing dispatch precedence between class and default methods with the same signature, do not alter semantics, all of which will be elaborated on later.

We propose an efficient, fully-automated, semanticspreserving refactoring approach that assists developers in taking advantage of enhanced interfaces. The approach, based on type constraints [67], works on large-scale projects with minimal intervention and features an extensive rule set, covering various corner-cases where default methods cannot be used. It identifies instances of the pattern and safely migrates class method implementations to interfaces as default methods.

The related PULL UP METHOD refactoring [78] safely moves methods from a subclass into a super class. Its goal is to solely reduce redundant code, whereas ours includes opening classes to inheritance, allowing classes to inherit multiple interface definitions, etc. Moreover, our approach deals with multiple inheritance, a more complicated type hierarchy involving interfaces, semantic differences due to class tiebreaking, and differences between class method headers and corresponding interface method declarations.

The refactoring approach is implemented as an open source Eclipse (http://eclipse.org) plug-in. The experimental evaluation used a set of 19 Java projects of varying size and domain with a total of $\sim 2.7$ million lines of code. Additionally, we submitted pull requests (patches) of the refactoring results to popular GitHub (http://github.com) repositories. Our study indicates that (i) the analysis cost is practical, with average running time of 0.115 seconds per input method and 0.144 seconds per thousand lines of code, (ii) the skeletal implementation pattern is commonly used in legacy Java software, and (iii) the proposed approach is useful in refactoring method implementations into default methods despite language restrictions. It also provides insight to language designers on how this new language constructs applies to existing software.

This work makes the following specific contributions:

Approach design. We present a novel automated refactoring approach for migration to Java 8 enhanced interface default methods. The approach infers which methods can be safely migrated to default methods via an exhaustive formulation of refactoring preconditions. We present new type constraints involving default methods and other modern Java constructs, as well as a scheme for semantics preservation in the context of tie breaking rules with classes. Furthermore, we identify all code changes required to perform the migration, including 
removal and replacement of skeletal implementation classes.

Implementation and experimental evaluation. The

approach is implemented as an open source Eclipse plug-in to ensure real-world applicability. A study on 19 Java programs indicates that the proposed techniques are effective and practical, and a pull request study demonstrates that the results are well-grounded. These results advance the state of the art in automated tool support for the evolution of legacy Java code to modern Java technologies.

\section{Motivating EXAMPLE}

In this section, we present an example that will be used throughout the paper to highlight the motivation of using default methods in legacy Java code, the refactoring challenges involved, and how our approach applies in particular situations.

Fig. 11 portrays a hypothetical collection type hierarchy snippet. Fig. 2 contains the corresponding UML class diagram. The hierarchy has been simplified for presentation, with only portions relevant to our refactoring shown, and illustrates specific issues that can arise with the refactoring. The original system (white space added for alignment) is pictured in Fig. 1(a), while Fig. 1(b) depicts the same system with several methods migrated to interfaces as default methods. Removed code is struck through, added code is underlined, and replaced code is both underlined and emphasized. Both systems are type-correct and semantically equivalent.

Several interfaces, including Collection and List, with the latter extending the former, meaning that any concrete class extending List must provide or inherit implementations of methods declared in both interfaces, are shown in Fig. 1(a) Note that, unlike classes, Java interfaces can extend multiple interfaces. Methods exist for determining a collection's size(), adding an element, whether it isEmpty(), its capacity (), and whether it is atcapacity (). Note that the abstract keyword is optional for interface methods.

Several methods also exist for Lists, including setting a List's size, removing its last element, adding an element (line 12, whose declaration overrides that of the super interface collection (commonly done for documentation [10]), replacing an element at a specified position, printing it to a specified stream, and copying it. Several methods are denoted as so-called optional operations as, e.g., not all list types may support modification. In such cases, implementers may throw an exception when these methods are invoked.

AbsList, an abstract class providing a skeletal implementation of a sequential, variable length List, is declared on line 18. Its purpose is to assist (concrete) classes in implementing the interface by declaring appropriate instance fields (line 20) and basic method implementations for the more primitive operations. Since it is abstract, it is not required to implement all interface methods. For the optional removelist (), the provided implementation (line 28) simply throws an UnsupportedOperationException. This way, concrete implementers extending AbsList that support element removal can override it with a working implementation, while others need not override it. The provided implementation of print() sends the standard string representation of the List to the stream. AbsLists are also Copiable (line 17), the provided implementation of which returns null instead of throwing an exception in the case that copying is unsupported.

A Queue interface snippet (line 36) contains two methods (one default) for offering and adding elements, respectively, with the former returning true if the operation was successful and false otherwise (e.g., if the queue is full) and the latter, written in terms of the former, throwing an exception.

Absqueue (line 40) provides a skeletal implementation of Queue 1 by extending AbsList, similar to an adapter pattern [11]. Unlike AbsList, it supplies functioning implementations for removelast() (useful for a pop operation, not shown) and add (), while also customizing the print () method. Another extension of AbsList, AbsUnmodList, which does not support the add operation, is declared on line 51. Absstack (line 55) specializes Abscontainer, and both it and Absset (line 58) implement Collection, providing isempty() implementations. Types implementing the Comparator interface (line 61) can order objects; DefaultComparator (line 63, of which cComparator (line 66 extends, supplies a basic ordering using hashes. Lastly, a main method (line 68) declares several concrete subclasses of various skeletal implementations of classes via anonymous inner classes (line 70).

Fig. 1(a) illustrates the skeletal implementation pattern, several drawbacks for which include:

Inheritance. Due to single-class inheritance restrictions, there is no clean way for AbsQueue to simultaneously benefit from AbsList and subclass another class. Moreover, Absqueue could not easily take advantage of skeletal implementations split over multiple abstract classes [12] $]^{2}$

Modularity. There is no syntactic path between List and AbsList, i.e., no syntax exists in List that refers to Abstist. In general, a whole program analysis may be required to find suitable skeletal implementers for interfaces as they may be split across different files and packages [5].

Bloated libraries. Abstist implements many of List's methods. This extra class can further complicate large libraries and make maintenance difficult. Additionally, method declarations are needed in both interfaces and classes to represent a single method and its default implementation.

Java 8 Default Methods. Default methods enable skeletal implementations in interfaces, thereby foregoing separate classes. Moreover, interface implementers need not search for separate skeletal implementations classes. Lastly, implementers can extend other classes, as well as inherit behaviors (but not state) from multiple interfaces, which can reduce the need for code duplication and forwarding methods [3].

Fig. 1(b) shows a refactored version of the running example, in which several skeletal implementations in classes have been migrated to interfaces as default methods. In the ab-

\footnotetext{
1 offer () uses a double to demonstrate issues related to strictfp.

${ }^{2}$ Implementers already extending a class can use the pattern via delegation to an internal class [4] at the expense of auxiliary forwarding code.
} 
interface Collection $<E>$,

int size ():

void add(E elem); // add to this collection (optional). boolean isEmpty ();

int capacity ();

abstract boolean atCapacity();

interface List $\langle\mathrm{E}>$ extends Collection $\langle\mathrm{E}\rangle$;

void setSize(int i) throws Exception;

void removelast(); // optional operation.

void add(E elem); // append to this list (optional).

void set (int i, @NamedArg (value="elementToSet") E e); void print (Printstream stream)

List $<\mathrm{E}>$ copy ();

interface Copiable $<\mathrm{E}>\{\mathrm{E}$ Copy ()$;\}$

abstract class AbsList $\langle E\rangle$ implements List $<E>$,

Copiable $<$ List $\langle\mathrm{E}\rangle>$ \{

Object[] elems; int size; // instance fields.

@Override public int size() \{return this.size;

dOverride public void setSize(int i) \{this.size $=i$;

(override public boolean isEmpty() \{return this.size ()==0; 2

QOverride public int capacity() \{return this.elems.length; 24

@Override public boolean atCapacity()

\{return this.size() == this.capacity ();

doverride public void removelast()

(throw new UnsupportedOperationException ();

@Override public void set (int $i$, @NamedArg (value="el") E e) \{this.elems [i] = e;

doverride public void print (Printstream out) \{out.println (this);

(Override public Abslist<E copy() \{

try \{return (AbsList $<E>$ ) this.clone ();

catch (Exception e) \{return null; \} \}

@Functionalinterface strictfp interface Queue $<E>$ \{

boolean offer( $\mathrm{E}$ elem);

default void add ( $E$ elem)

if (!offer(elem)) throw new RuntimeException ("full"); \}

abstract class AbsQueue $<E>$ extends AbsList $<E>$ implements

Queue $<\mathrm{E}>\{$

@Override public void removelast()

\{if (! isEmpty ()) this.setSize(this.size ()-1);

eOverride public void add (E elem) \{// resize if necessary

this.set (this.size(), elem); this.setsize(this.size ()+1); $\}_{45}$

@Override public void print (PrintStream out) \{super.print(out); out.println("Printing queue ..."); \} 47

QOverride @Managedoperation public boolean offer( $\mathrm{E}$ elem) $\left\{\begin{array}{l}48 \\ 48\end{array}\right.$ if (size() $+1.0<$ capacity ()$)$ \{add(elem); return true; ${ }_{49}$ else return false; \}

abstract class AbsUnmodList $\angle \mathrm{E}>$ extends AbsList $<\mathrm{E}>$

@Override public void add (E elem)

\{throw new UnsupportedoperationException (); \}

abstract class AbsContainer $\{/ * \ldots * /\}$

abstract class AbsStack $<\mathrm{E}>$ extends AbsContainer implements

Collection $<\mathrm{E}>$

doverride public boolean isEmpty() \{return this.size ()==0; 56 abstract class AbsSet $\langle\mathrm{E}\rangle$ implements Collection $\langle\mathrm{E}\rangle$ \{

(Override public boolean isEmpty()

int size = this.size (); return size $==0$; \}

interface Comparator $\langle\mathrm{T}\rangle$ int compare $(\mathrm{T} \circ 1, \mathrm{~T} \circ 2)$;

abstract class DefaultComparator $\left\langle\mathrm{T}>\right.$ implements Comparator $<\mathrm{T}>f_{6}$

QOverride public int compare ( $\mathrm{T}$ o1, $\mathrm{T}$ 02) (

return Objects.hashCode (o1)-Objects.hashCode (o2); \}

class CComparator $<\mathrm{T}>$ extends DefaultComparator $<\mathrm{T}>\{/ \star \ldots \star /\}$

class Main

public static void main(String [] args)

Absqueue<Integer $>$ queue $1=$ new AbsQueue<Integer $>()\{\}$;

queue1. removeLast ();

Abslist<Integer $>$ queue 2 = queue1. $\operatorname{copy}()$;

Queue<String $>$ queue $3=($ s) $->$ true

assert (new AbsUnmodList<String $>($ ) \{\}. isEmpty ()),

Absistack stack $=/ / \ldots$

Collection $\mathrm{col}=$ stack

Abscontainer container = stack; $\}$

(a) Using abstract skeletal implementation classes to ease interface implementation. interface Collection $<E>$

int size():

void add(E elem); // add to this collection (optional). default boolean isEmpty () \{return this.size ()$==0$;

int capacity ();

abstract default boolean atCapacity()

\{return this.size ()$==$ this.capacity ()$;\}\}$

interface List $<\mathrm{E}>$ extends Collection $\langle\mathrm{E}>$ (

void setSize(int i) throws Exception;

default void removelast() // optional operation.

\{throw new UnsupportedOperationException ();

default void add(E elem) // append to this list (optional). \{throw new UnsupportedOperationException ();

void set (int i, @NamedArg(value="elementToSet") E e);

default void print (PrintStream out) \{out.println(this);

List $<\mathrm{E}>$ copy ();

interface Copiable $<E>$ E $\operatorname{Copy}() ;\}$

abstract class AbsList $<E>$ implements List $<E>$, Copiable<List $\langle\mathrm{E}\rangle>$ \{

object[] elems; int size; // instance fields.

@Override public int size() \{return this.size;

@Override public void setSize(int i) \{this.size = i;

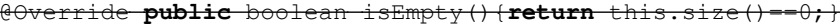

a Override public int capacity() \{return this.elems.length;

everride public boolean atcapacity ()

treturn this.size () == this.capacity ();

aride publie to

throw new UnsupportedoperationException();

@override public void set (int $i$, (NamedArg (value="el") E e) \{this.elems $[i]=e$;

Q Override public void print (Printstream out)

tout.print ln(this);

QOverride public AbsList<E $>$ copy() (

try return (AbsList $<E>$ ) this.clone ();

catch (Exception e) \{return null; \} \}

@Functionalinterface strictfp interface Queue $<E>$ \{

boolean offer( $\mathrm{E}$ elem);

default void add ( $E$ elem)

\{if (!offer(elem)) throw new RuntimeException("full"); \} \}

abstract class AbsQueue $\langle\mathrm{E}\rangle$ extends AbsList $\langle\mathrm{E}>$ implements Queve $<\mathrm{E}>$,

QOverride public void removelast()

\{if (!isEmpty()) this.setSize(this.size()-1); \}

@Override public void add(E elem) \{ // resize if necessary. this.set (this.size(), elem); this.setSize (this.size ()+1); \}

@Override public void print (Printstream out) \{super.print (out); out.println("Printing queue ...");

QOverride @Managedoperation public boolean offer(E elem) if (size ()$+1.0<\operatorname{capacity}()) \quad$ add (elem); return true; else return false; \}

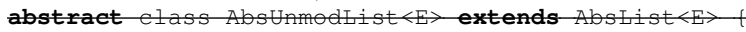

eOverride public void add ( $\mathrm{E}$ elem)

(throw new Unsupportedoperationexception (); \})

abstract class AbsContainer $\{/ \star \ldots * /\}$

abstract class AbsStack $\langle E\rangle$ extends AbsContainer implements Collection $\langle\mathrm{E}\rangle$.

everide publie bolean is y () (return this.size ()=-0;)

abstract class AbsSet $\langle\mathrm{E}>$ implements Collection $\langle\mathrm{E}>$ \{

QOverride public boolean isEmpty()

int size $=$ this.size () ; return size $==0$; $\}$ \}

interface Comparator $\langle\mathrm{T}>$ default int compare $(\mathrm{T} 01, \mathrm{~T} \circ 2$ )

\{return objects.hashCode (01)-objects.hashCode $(02) ;\}$ \}

abstract class DefaultComparator $<T>$ implements Comparator $<T>t$ over publie int (T $01, T-O Z)$

return objects. hashcode (01) Objects.hashcode $(02) ;\}\}$

class CComparator $\langle\mathrm{T}\rangle$ implements DefaultComparator $\langle\mathrm{T}\rangle\{/ \star \ldots \star /\}$

class Main \{

public static void main(String[] args)

AbsQueue $<$ Integer $>$ queue $1=$ new AbsQueue $<$ Integer $>()\{\}$; queue1.removeLast ();

AbsList<Integer $>$ queue $2=$ queue 1 . copy () ;

Queue<String $>$ queue $3=(\mathrm{s})->$ true;

assert (new Abs List<String $>()\{\}$. isEmpty ())

AbsStack stack $=/ 1$. .

Collection $\operatorname{col}=$ stack;

Abscontainer container = stack; $\}$

(b) Improvements after our refactoring is applied.

Fig. 1. A running example of a collection type hierarchy (inspired by [7[9]). 


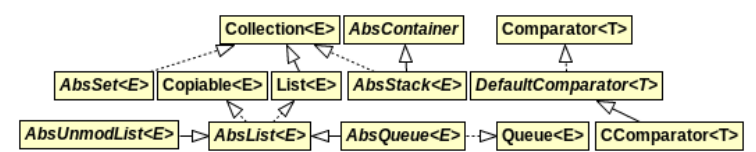

Fig. 2. Collection type hierarchy UML class diagram.

stract classes, the migrated methods were completely removed, including the method header and eoverride annotations ${ }^{3}$ For each migrated method, the default keyword was inserted before the method return type and the delimiter was replaced with the body from the abstract class. In the case of collection.atCapacity (), the abstract keyword on the method was removed (line 6) as the method is now concrete. For List.print (), the parameter name was altered to match that of the migrated method implementation $4^{4}$

Now, developers considering implementing the interfaces can clearly recognize List.removelast() as optional and what should happen when it is not implemented. Classes can inherit the default method collection.isEmpty () without the need for finding and inheriting from a separate class or duplicating existing or writing new forwarding code.

AbsUnmodList and DefaultComparator were completely removed in Fig. 1(b) CComprator now implements Comparator rather than extending DefaultComparator (line 66) and inherits the default implementation of compare() from the interface, freeing it to extend other classes. Similar transformations are also required for classes extending AbsUnmodList but are slightly different depending on context. For example, the anonymous inner class (AIC) declared on line 73 now uses the interface rather than the class in its constructor call, making developer intent more explicit.

\section{Problem AnAlysis}

To highlight refactoring challenges, we examine Fig. 1(a) more carefully, revealing the following:

- In Abstist, methods isEmpty(), atCapacity(), removelast(), print(), in AbsUnmodList, method add(), in Absstack, method isEmpty(), and in Defaultcomparator, method compare() can be migrated without affecting type-correctness and program semantics as classes now inherit them as default methods. The transformation also occurs unambiguously; each method has a well-defined, unique "destination" interface. Several methods are migrated to "indirect" interfaces, i.e., those not explicitly implemented by their declaring class but rather a super class up the type hierarchy.

- The target method Collection.isEmpty() has multiple source methods: AbsList.isEmpty(), AbsStack.isEmpty (), AbsSet.isEmpty(). Only the first two were migrated due to common implementations ${ }^{5}$

\footnotetext{
${ }^{3}$ The @Override annotation is not carried over to the default method since the method body is no longer in an overriding relationship.

${ }^{4}$ Alternatively, each parameter reference could have been modified to match the interface parameter, however, changing only one location is less invasive.

${ }^{5} \mathrm{Abs}$ Set. isEmpty () contains an unjustifiably different implementation to demonstrate issues that arise in complicated type hierarchies.
}

- Methods size(), setSize(), capacity(), and set() in Abstist cannot be migrated because they access instance fields of the receiver, which cannot be migrated. Also, offer() in AbsQueue cannot be migrated because size() and capacity () are not declared in Queue. Nevertheless, exploring composite refactorings that may compensate in certain cases is an area for future work.

- Methods add() in AbsunmodList and copy() in AbsList have multiple interface abstract target methods for which they provide implementations. Specifically, since AbsUnmodList extends AbsList, AbsList implements List, and List extends Collection, AbsUnmodList provides an add() implementation for methods in both interfaces. The migration must be to List, otherwise, migrating to collection would result in a compilation error at line 73 because the (new) default implementation would be squelched by the corresponding abstract method in List further down the hierarchy. In the case of copy (), since AbsList implements multiple interfaces, the ambiguity occurs across the type hierarchy as both the List and Copiable interfaces declare a copy () method.

- Method setSize() in AbsList also cannot be migrated to List as Abstist. setSize() does not declare that an exception is thrown, while List.setSize() does (note that this is type-correct). Migrating it would result in a compiletime error at the call at line 43 because removelast () does not deal with the declared thrown exception. Also, in the same class, copy () cannot be migrated to List as doing so would result in a compile-time error at line 71 because queue1.copy () would return List, which is not (a subtype of) Abstist. The same method could also not be migrated to copiable because the returned value of AbsList $<E>$ (line 34) is not type-compatible with the generic return type $\mathrm{E}$ from Copiable.

- copy () in AbsList also cannot be migrated because it calls clone (), which is a protected method of object. While interfaces do not extend Object, they implicitly declare abstract methods for (only) each public object method, which does not include clone () [13, Ch. 9.2]. Moreover, print () in Absqueue cannot be migrated as it contains an unqualified reference to super, which is disallowed in interfaces to prevent the diamond problem [14[15].

- Note that Queue is functional (via the annotation at line 36, meaning that lambda expressions can be used to represent types implementing the interface. An interface may also be effectively functional, i.e., an annotation is not required to instantiate an interface using a lambda expression. It is required that functional interfaces have exactly one abstract method so that it is not ambiguous as to which method is invoked when the lambda expression is evaluated [13 Ch. 9.8]. As such, migrating offer() from Absqueue to Queue would result in a type-incorrect program because Queue would no longer have any abstract methods, thus invalidating the lambda expression on line 72 .

- Method add() in Absqueue would be otherwise fine to migrate to List, however, doing so would produce a type- 
incorrect program as AbsQueue would "inherit" multiple interface methods, i.e., add () from List and Queue. Such a situation is disallowed by Java's typing rules ${ }^{6}$ Note that this situation would also arise even if Queue.add () were default. Furthermore, the same problem would emerge in Absqueue had add () been defined in one of its subclasses.

- Queue is strictfp (line 36), meaning all calculations within its methods use strict floating-point math. While not related to type-correctness, migrating offer() from AbsQueue (line 48) to Queue could possibly alter semantics as it is not strictfp. The modifier can also be used at the method level, where a similar mismatch can occur. Additionally, there is an difference in annotation types between the two methods, possibly affecting processing frameworks like dependency injection. set () in AbsList (line 29) also has an annotation difference with the method in List (line 14), except here the difference is in the annotation value.

- Migrating method removelast () in Absqueue (line 42) to List does not result in any type-incorrect code, however, semantics would be altered as the run time target of the call at line 70 would change to the method in AbsList. This is because Absqueue would inherit two different different versions of removelast (), one from class AbsList and the other from interface List. In this situation, classes take precedence over interfaces. Moreover, we would have this problem had the conflicting method come from a directly implemented interface rather than from the class hierarchy.

- As a result of the refactoring, AbsUnmodList (line 51) is now empty, i.e., no methods, fields, or inner types remain. As such, it can be removed and the reference at line 73 can be replaced with its super class (see Fig. 1(b)).

- Although Absstack (line 55) is now also empty, it cannot be removed because replacing the reference at line 74 with its super class would produce an error at line 75 as it does not implement collection. Replacing it with the implemented interface would also cause an error at line 76 as Abscontainer is not (a super type of) collection.

- DefaultComparator (line 63) is now empty as well and can be removed with the reference at line 66 being replaced with the implemented interface and changing extends to implements. However, we must ensure that subclasses like CComparator do not contain references to super that are not type-compatible with their new super class object.

\section{Migration ApProACH}

Assumptions. Our approach operates on a closed-world assumption that assumes full accessibility to all source code that could possibly affect or be affected by the refactoring. We also assume that method calls and references to fields and types can be statically resolved and that the original program successfully compiles under a Java 8 compiler. $\$[\mathrm{~V}$ discusses how we relax several of these in our implementation.

\footnotetext{
${ }^{6}$ In this situation, the interface method to inherit must be explicitly selected by overriding the method, e.g., in AbsQueue, by calling one of them, e.g., List.super.add (). However, doing so would be in conflict with our goals as we would be delegating the class method to the default one.
}

Top-level Processing. Our input is concrete instance methods declared in abstract classes that implement at least one interface. $\$ \sqrt{\mathrm{V}}$ discusses how using only abstract classes increases the likelihood that these methods would make suitable default methods; \$VII considers pattern variations. Each method must have a body and not be final nor synchronized, as such methods are disallowed in interfaces [13].

Type Constraints. To determine whether migrating a method to an interface as a default method results in a type-correct program, we build upon an existing framework of type constraints [67]. For each program element, type constraints denote the subtyping relationships that must hold between corresponding expressions for that portion of the system to be considered well-typed. Thus, a complete program is typecorrect if all constraints implied by all program elements hold.

Notation and Terminology: In line with [7], we will use the term declaration element to represent declarations of local variables, method parameters, fields, and method return types. We will use $v$ to denote variables, $M$ for methods, $F$ for fields, $C$ for classes, $I$ for interfaces, $E x$ for exceptions, and $T$ types (either a class, interface, or enum). We should note that the symbol $M$ represents a method along with its signature, return type, and exception information, as well as a reference to its declaring type. Likewise, $F$ and $C$ denote a field and a type, respectively, along with its name, type in which it is declared, and its declaring type in the case of fields. Furthermore, we will use $E$ to represent an expression or declaration element in a specific point in the program's abstract syntax tree. Type information regarding expressions and declaration elements are assumed to be available from the compiler.

Next, we define notions of virtual methods and root definitions, taken directly from [7] for expressing the type constraint for method overriding. A method $M$ is virtual if $M$ is not a constructor, $M$ is not private and $M$ is not static. Def.[1]defines the concept of overriding 7

Definition 1 (overriding). A virtual method $M$ in type $C$ overrides a virtual method $M^{\prime}$ in type $B$ if $M$ and $M^{\prime}$ have identical signatures and $C$ is equal to $B$ or $C$ is a subtype of $B .^{8}$ In this case, we also say that $M^{\prime}$ is overridden by $M$.

Def. 2 pertains to the concept of root definitions. For a method $M, \operatorname{RootDefs}(M)$ is the set containing the most general methods in the type hierarchy that are overridden by $M$. Using our motivating example in Fig. 1. RootDefs(Absqueue.add()) = $\{$ Queue.add (), Collection. add ()\}.

Definition 2 (root definitions). Let $M$ be a method. Define:

RootDefs $(M)=\left\{M^{\prime} \mid M\right.$ overrides $M^{\prime}$, and there exists no $M^{\prime \prime}\left(M^{\prime \prime} \neq M^{\prime}\right)$ such that $M^{\prime}$ overrides $\left.M^{\prime \prime}\right\}$

Def. 3 presents a new notion of exception handling specific to our paper, particularly helpful in dealing with ex-

\footnotetext{
${ }^{7}$ This definition expresses that a virtual method overrides itself.

${ }^{8} \mathrm{We}$ ignore access rights as interface methods are always public, whose visibility cannot be reduced. Throws clauses are a topic for future work.
} 


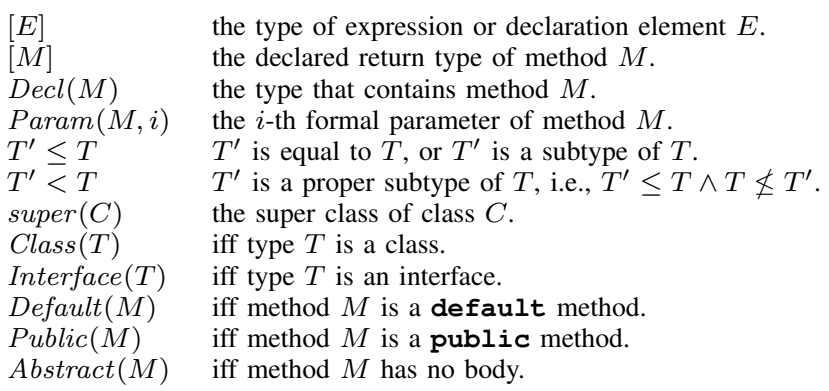

Fig. 3. Type constraint notation (inspired by [7]).

ception throws clause differences between source and target methods. For a given method call expression E.m(...), Handle $(\operatorname{E.m}(\ldots))$ is the set of checked exceptions either thrown (declared by the enclosing method) or caught (by a surrounding try-catch block) at the expression. It is computed by intersecting the checked exceptions declared as being thrown by the compile-time target of $\operatorname{E.m}(\ldots)$ with those appearing in corresponding catch blocks surrounding the call.

Definition 3 (handled exceptions). Let E.m(...) be a method call expression. Define:

$$
\begin{aligned}
\text { Handle }(E . m(\ldots))= & \left\{E x_{h} \mid E x_{h}\right. \text { is a checked exception } \\
& \text { either thrown or caught at E.m }(\ldots)\}
\end{aligned}
$$

Another concept specific to our paper is that of a functional interface, captured by Def. 4. It is useful in preventing existing lambda expressions from being invalidated.

Definition 4 (functional). An interface $I$ is functional iff $I$ is annotated with eFunctional Interface or if there exists a lambda expression implementing I, in which case I has exactly one abstract method (effectively functional).

Fig. 3 portrays the notation, including several helper predicates, we will use to describe type constraints. Let $\alpha$ be a constraint variable, which can be a type constant $T,[E]$, i.e., the type of an expression or declaration element $E$, $\operatorname{Decl}(M)$, i.e., the type declaring $\operatorname{method} M$, or $\operatorname{Decl}(F)$, the type declaring field $F$. Then, a type constraint can be one of: (i) $\alpha_{i} \triangleq \alpha_{j}$, i.e., $\alpha_{i}$ is defined to be the same as $\alpha_{j}$, (ii) $\alpha_{i} \leq \alpha_{j}$, i.e., $\alpha_{i}$ must be equal to or a subtype of $\alpha_{j}$, (iii) $\alpha_{i}=\alpha_{j}$, i.e., $\alpha_{i} \leq \alpha_{j} \wedge \alpha_{j} \leq \alpha_{i}$, and (iv) $\alpha_{i}<\alpha_{j}$, i.e., $\alpha_{i} \leq \alpha_{j} \wedge \alpha_{j} \not \alpha_{i}$. Note that if $T^{\prime}$ is a class and $T$ is an interface, $T^{\prime}<T$ means that $T^{\prime}$ implements $T$. If both $T^{\prime}$ and $T$ are interfaces, then $T^{\prime}<T$ means that $T^{\prime}$ extends $T$.

Inferring Type Constraints: Fig. 4 shows several rules for generating type constraints for many Java constructs. Constraints (5), (12)-(14), (16)-(18), (25), and (26) are new. The remaining are from [7]. Of these, due to space limitations, we only show those that are relevant here. Constraints 21- 24 define the types of declaration elements, while constraints 25] and (26) are for default method migration.

As an example, for a virtual method call $\operatorname{E.m}\left(E_{1}, \ldots, E_{n}\right)$ that statically resolves to a method $M$, by rule (2), the type of the method call expression is that of $M$ 's return type. It is also required by rule (3) that each method argument $E_{i}$ be a subtype or equal to each method parameter $\operatorname{Param}(M, i)$. Furthermore, the type of the receiver expression $E$ must either declare or inherit the called method, expressed by rule (4) using Def. 2. For each of the thrown exceptions $M$ declares, there must exist an exception handled at the call such that the thrown exception is a subtype or equal to the handled exception, expressed by our new rule (5) and Def. 3.

For convenience, let us combine rules 9 and 10 into a single "nearly" overriding relation NOverrides $\left(M^{\prime}, M\right) \equiv$ $\left[\operatorname{Param}\left(M^{\prime}, i\right)\right]=[\operatorname{Param}(M, i)] \wedge\left[M^{\prime}\right] \leq[M]$, which is a looser version of the constraints for method overriding, not requiring any relationship between the declaring types of $M$ and $M^{\prime}$. Rule (13) expresses that interfaces, unlike classes and enums, do not inherit from object (cf. rule (12)), although they are subtypes of object [16]. They do, however, have public methods $(\operatorname{Public}(M))$ from object $(\operatorname{Decl}(M) \triangleq$ java.lang. Ob ject) available to them $\left(\operatorname{Decl}\left(M^{\prime}\right) \triangleq I \wedge\right.$ NOverrides $\left(M^{\prime}, M\right)$ ), i.e., they can be called whether or not they are explicitly declared by the interface [13, Ch. 9.2].

Rule (14) ensures that functional interfaces (see Def. 4) declare exactly one abstract method. For types other than interfaces, the type of super in method $M$ is defined to be that of the super class of $M$ (rule (16)). Otherwise, an unqualified super reference is undefined. For interface qualified super references where the declaring type of $M$ implements the referenced interface, the type is defined to be that interface (rule (17)). Rule (18) defines the type of anonymous inner class (AIC) creation expressions.

Rule 25 is enforced for each method declared in an interface. For each method $M$ declared in interface $I$, the rule applies if there is an unrelated interface $J$, another method $M^{\prime}$ declared either in $J$ or inherited by $J$ such that both $M^{\prime}$ and $M$ have similar method signatures (NOverrides $\left(M^{\prime}, M\right)$ ) and at least one of them is a default method. In these cases, for all classes $C$ such that $C$ implements both $I$ and $J$, there must exist a public class method $M^{\prime \prime}$ disjoint from $M^{\prime}$ and $M$ such that $C$ either declares or inherits $M^{\prime \prime}$, and $M^{\prime \prime}$ either implements or overrides at least one of the corresponding methods in $I$ and $J$. If rule 25] is not satisfied, the underlying program will not be type-correct as $C$ and its subtypes will "inherit" at least two different versions of the same interface method. Note that this problem arises even if not all the inherited methods are default. An example application of this and the following rule is provided in the next subsection.

Rule 26) ensures that any concrete type $T$ implementing an interface $I$ that declares a method $M$ defines or inherits an implementation for $M$. The clause $T \leq \operatorname{Decl}\left(M^{\prime}\right) \wedge$ NOverrides $\left(M^{\prime}, M\right)$ is equivalent to the constraints for method overriding. It states that there must exist such a method $M^{\prime}$ with a body (i.e., $\neg \operatorname{Abstract}\left(M^{\prime}\right)$ ) and, moreover, that there are no methods $M^{\prime \prime}$ between the two (i.e., $\left.T<\operatorname{Decl}\left(M^{\prime \prime}\right)<\operatorname{Decl}\left(M^{\prime}\right)\right)$ in the type hierarchy that is abstract, thus squelching the inherited implementation.

Checking Migration Preconditions. We will apply the type constraints to determine if migrating a class method to its 


\begin{tabular}{|c|c|c|}
\hline program construct & implied type constraint(s) & \\
\hline assignment $E_{i}=E_{j}$ & {$\left[E_{j}\right] \leq\left[E_{i}\right]$} & $(1)$ \\
\hline $\begin{array}{l}\text { method call } \\
E \cdot m\left(E_{1}, \ldots, E_{n}\right) \\
\text { to a virtual method } M \\
\text { (throwing exceptions } \\
\left.E x_{t 1}, \ldots, E x_{t j}\right)\end{array}$ & $\begin{array}{c}{\left[E \cdot m\left(E_{1}, \ldots, E_{n}\right)\right] \triangleq[M]} \\
{\left[E_{i}\right] \leq[\operatorname{Param}(M, i)]} \\
{[E] \leq \operatorname{Decl}\left(M_{1}\right) \vee \cdots \vee[E] \leq \operatorname{Decl}\left(M_{k}\right)} \\
\text { where } \operatorname{RootDefs}(M)=\left\{M_{1}, \ldots, M_{k}\right\} \\
\forall \operatorname{Ex} x_{t} \in\left\{\operatorname{Ex}_{t 1}, \ldots, E x_{t}\right\} \\
\exists E x_{h} \in \operatorname{Handle}\left(E \cdot m\left(E_{1}, \ldots, E_{n}\right)\right)\left[\left[E x_{t}\right] \leq\left[E x_{h}\right]\right]\end{array}$ & $\begin{array}{l}\text { (2) } \\
(3) \\
(4) \\
(5)\end{array}$ \\
\hline access $E . f$ to field $F$ & $\begin{array}{l}{[E . f] \triangleq[F]} \\
{[E] \leq \operatorname{Decl}(F)}\end{array}$ & $\begin{array}{l}(6) \\
(7)\end{array}$ \\
\hline return $E$ in method $M$ & {$[E] \leq[M]$} & $(8)$ \\
\hline $\begin{array}{l}M^{\prime} \text { overrides } M \\
\quad M^{\prime} \neq M\end{array}$ & $\begin{aligned} {\left[\operatorname{Param}\left(M^{\prime}, i\right)\right] } & =[\operatorname{Param}(M, i)] \\
{\left[M^{\prime}\right] } & \leq[M] \\
\operatorname{Decl}\left(M^{\prime}\right) & <\operatorname{Decl}(M)\end{aligned}$ & $\begin{aligned}(9) \\
(10) \\
(11)\end{aligned}$ \\
\hline for every class (and enum) $C$ & $C \leq$ java.lang. Ob ject & $(12)$ \\
\hline for every interface $I$ & $\begin{array}{r}I \not \leq \text { java.lang.Object } \wedge \forall M[\operatorname{Decl}(M) \triangleq \text { java.lang.Object } \wedge \\
\left.\operatorname{Public}(M) \Longrightarrow \exists M^{\prime}\left[\operatorname{Decl}\left(M^{\prime}\right) \triangleq I \wedge \operatorname{NOverrides}\left(M^{\prime}, M\right)\right]\right]\end{array}$ & $(13)$ \\
\hline for every functional interface $I$ & $\begin{aligned} \exists M[\operatorname{Decl}(M) & \triangleq I \wedge \operatorname{Abstract}(M) \\
& \left.\wedge \forall M^{\prime}\left[\operatorname{Decl}\left(M^{\prime}\right) \triangleq I \wedge M^{\prime} \neq M \Longrightarrow \neg \operatorname{Abstract}\left(M^{\prime}\right)\right]\right]\end{aligned}$ & $(14)$ \\
\hline implicit declaration of this in method $M$ & this $] \triangleq \operatorname{Decl}(M)$ & $(15)$ \\
\hline implicit declaration of super in method $M$ & $\neg \operatorname{Interface}(\operatorname{Decl}(M)) \Longrightarrow[$ super $] \triangleq \operatorname{super}(\operatorname{Decl}(M))$ & $(16)$ \\
\hline implicit declaration of I. super in method $M$ & $\operatorname{Decl}(M)<I \Longrightarrow[$ I. super $] \triangleq I$ & $(17)$ \\
\hline expression new $T\left(E_{1}, \ldots, E_{n}\right) \ldots$ & {$\left[\right.$ new $\left.T\left(E_{1}, \ldots, E_{n}\right) \ldots\right] \leq[T]$} & $(18)$ \\
\hline declaration of method $M($ declared in type $T)$ & $\operatorname{Decl}(M) \triangleq T$ & $(19)$ \\
\hline declaration of field $F$ (declared in type $T$ ) & $\operatorname{Decl}(F) \triangleq T$ & $(20)$ \\
\hline explicit declaration of variable or method parameter $T v$ & {$[v] \triangleq T$} & $(21)$ \\
\hline declaration of method $M$ with return type $T$ & {$[M] \triangleq T$} & $(22)$ \\
\hline declaration of field $F$ with type $T$ & {$[F] \triangleq T$} & $(23)$ \\
\hline cast $(T) E$ & {$[(T) E] \triangleq T$} & $(24)$ \\
\hline declaration of method $M$ declared in interface $I$ & $\begin{array}{r}\exists J, M^{\prime}\left[\text { Interface }(J) \wedge J \nless \leq I \wedge I \nless J \wedge J \leq \operatorname{Decl}\left(M^{\prime}\right)\right. \\
\left.\wedge N \text { Overrides }\left(M^{\prime}, M\right) \wedge\left(\operatorname{Default}\left(M^{\prime}\right) \vee \operatorname{Default}(M)\right)\right] \\
\Longrightarrow \forall C \mid \operatorname{Class}(C) \wedge C<I \wedge C<J\left[\exists M^{\prime \prime}\left[M^{\prime \prime} \neq M^{\prime} \wedge M^{\prime \prime} \neq M\right.\right. \\
\wedge \operatorname{Class}\left(\operatorname{Decl}\left(M^{\prime \prime}\right)\right) \wedge C \leq \operatorname{Decl}\left(M^{\prime \prime}\right) \wedge \operatorname{Public}\left(M^{\prime \prime}\right) \\
\left.\left.\wedge \operatorname{NOverrides}\left(M^{\prime \prime}, M^{\prime}\right)\right]\right]\end{array}$ & $(25)$ \\
\hline $\begin{array}{c}\text { declaration of concrete type } T \text { implementing interface } I \\
\text { declaring method } M\end{array}$ & $\begin{array}{l}\exists M^{\prime}\left[T \leq \operatorname{Decl}\left(M^{\prime}\right) \wedge N \text { Overrides }\left(M, M^{\prime}\right)\right. \\
\wedge \neg \operatorname{Abstract}\left(M^{\prime}\right) \wedge \forall M^{\prime \prime}\left[T<\operatorname{Decl}\left(M^{\prime \prime}\right)<\operatorname{Decl}\left(M^{\prime}\right) \wedge\right. \\
\left.\left.N \text { Overrides }\left(M^{\prime \prime}, M^{\prime}\right) \Longrightarrow \neg \operatorname{Abstract}\left(M^{\prime \prime}\right)\right]\right]\end{array}$ & $(26)$ \\
\hline
\end{tabular}

Fig. 4. Type constraints for a subset of core Java features.

implementing method in an interface as a default method affects type-correctness using Fig. 1(a).

- Migrating Abstist.size() to the method it implements, i.e., collection.size() as a default method implies that $[$ this] = Collection, violating constraint (7) that [this] $\leq$ [AbsList]. Migrating AbsQueue.offer() to Queue implies that Queue $\leq$ collection, violating constraint (4) that [this] $\leq \operatorname{Decl}$ (Collection. capacity ()).

- Migrating

Collection AbsUnmodList.add ()

(Col) implies

to

AbsunmodList $<.>()\{\} \leq \operatorname{Decl}(\operatorname{Col} \cdot \operatorname{add}()) \wedge$ NOverrides(AbsUnmodList $<. .>()\{\}$, Col.add ()) $\wedge$ $\neg$ Abstract $(\mathrm{Col}$. add ()) violating constraint 26 because AbsUnmodList $<\ldots>()\{\}<$ $\operatorname{Decl}($ List.add ()) $<\operatorname{Decl}(\operatorname{Col} \cdot \operatorname{add}()) \wedge$ NOverrides (List.add (), Col.add ())

Abstract(List.add()). Migrating to List, however, does not violate the type constraint. The ambiguity arising from identifying the destination interface for Abstist.copy () is not as easily resolvable. We discuss handling ambiguity across the type hierarchy further below.

- Migrating Abstist.setSize() to List implies that the method now throws an Exception, violating constraint (5) as Handle(this.setsize(this.size()-1)) $=\varnothing$. Migrating AbsList.copy() to Copiable $<\mathrm{E}>$ implies that $[($ AbsList $<E>)$ this.clone ()$]=$ AbsList $<E>$, which violates constraint (8) that $[\mathrm{AbsList}<\mathrm{E}>] \leq[\mathrm{E}]$.

- Migrating AbsList.copy() to List implies that [this] $=$ [List], violating constraint (4) that [this] $\leq \operatorname{Decl}$ (Object.clone()) as List $\$$ object (cf. rule (13)). By rule (13), clone() is not available to interfaces as it is protected. By migrating Absqueue.print () to List, the type of the unqualified super reference would be undefined by rule (16).

- Migrating Absqueue.offer() to Queue implies that Queue has no abstract methods, violating constraint (14).

- Migrating Absqueue.add() to either List or Collection implies that AbsQueue now inherits multiple add () interface methods, one of which contains a body, and does not override the method, violating constraint 25]. 
Note that the constraint would also have been violated had add () been defined in an AbsQueue subclass.

- While not related to type-correctness, migrating AbsQueue.offer() to Queue may alter the semantics of floating-point calculations. To solve this, we implicitly propagate all type-level strictfp modifiers to all constituent methods and require that the source method modifier match that of the target. A similar technique is employed for annotations but if an annotation value is present, as is the case with migrating AbsList.set () to List, the values, which are always statically available, must also match. The doverrides annotation is excluded as it is removed. Type constraints are not used for these cases because they are not related to type-correctness but rather semantics-preservation. Since type constraints express relationships over only a single program versions, they are not typically used to preserve semantics [7].

- Migrating Absqueue.removelast () to List also does not raise any type-correctness issues but does alter semantics. To prevent this, we disallow from migration methods that override methods (using Def. 1) in both classes and interfaces. In this way, the migrated method, that would now reside in an interface rather than a class, will never lose in a tie to a class method with a similar signature.

- Replacing AbsStack with its super class AbsContainer on line 74 implies [stack] $\triangleq$ Abscontainer by rule (21), which violates constraint (1) that [Abscontainer $] \leq$ [Collection], generated from line 75. Replacing Absstack with its implemented interface collection instead also violates the constraint that Collection $\leq$ Abscontainer, generated from line 76 Cases where the class to remove implements multiple interfaces is discussed further below.

- By removing the explicit super class of CComparator, the type of super in any of its methods changes from DefaultComparator to object per rule (16). Any uses of super in CComparator to reference elements of DefaultComparator would violate type constraints.

Ambiguous Destination Interfaces. When the destination interface is ambiguous, if the ambiguity lies up the type hierarchy, we select the "closest" method to the source method up the hierarchy, which is akin to selecting a method not violating constraint 26. When the ambiguity is across the hierarchy, more input is needed. Currently, such methods fail preconditions to facilitate full automation, however, in our experiments, this accounted for only $0.87 \%$ of failures. Nevertheless, $\$$ VII explores dealing with such ambiguity.

Ambiguous Substitutable Interfaces. When classes become empty, we determine the type, which can be specific to each reference, to use in replacing references. Where the class has no (explicit) super class, one of the implemented interfaces is used. If multiple valid interfaces, i.e., those that do not violate any type constraints when substituted, are available at a particular reference expression, choosing any of them suffices.

Target Methods with Multiple Source Methods. As seen with Collection.isEmpty(), there may not be a one-

\begin{tabular}{lllllllll}
\hline subject & $\mathrm{KL}$ & $\mathrm{KM}$ & $\mathrm{cnds}$ & $\mathrm{dflts}$ & $\mathrm{fps}$ & $\delta$ & $-\delta$ & $\mathrm{tm}(\mathrm{s})$ \\
\hline ArtOfIllusion & 118 & 6.94 & 16 & 1 & 34 & 1 & 0 & 3.65 \\
Azureus & 599 & 3.98 & 747 & 116 & 1366 & 31 & 2 & 61.83 \\
Colt & 36 & 3.77 & 69 & 4 & 140 & 3 & 0 & 6.76 \\
elasticsearch & 585 & 47.87 & 339 & 69 & 644 & 21 & 4 & 83.30 \\
Java8 & 291 & 30.99 & 299 & 93 & 775 & 25 & 10 & 64.66 \\
JavaPush & 6 & 0.77 & 1 & 0 & 4 & 0 & 0 & 1.02 \\
JGraph & 13 & 1.47 & 16 & 2 & 21 & 1 & 0 & 3.12 \\
JHotDraw & 32 & 3.60 & 181 & 46 & 282 & 8 & 0 & 7.75 \\
JUnit & 26 & 3.58 & 9 & 0 & 25 & 0 & 0 & 0.79 \\
MWDumper & 5 & 0.40 & 11 & 0 & 24 & 0 & 0 & 0.29 \\
osgi & 18 & 1.81 & 13 & 3 & 11 & 2 & 0 & 0.76 \\
rdp4j & 2 & 0.26 & 10 & 8 & 2 & 1 & 0 & 1.10 \\
spring & 506 & 53.51 & 776 & 150 & 1459 & 50 & 13 & 91.68 \\
Tomcat & 176 & 16.15 & 233 & 31 & 399 & 13 & 0 & 13.81 \\
verbose & 4 & 0.55 & 1 & 0 & 1 & 0 & 0 & 0.55 \\
VietPad & 11 & 0.58 & 15 & 0 & 26 & 0 & 0 & 0.36 \\
Violet & 27 & 2.06 & 104 & 40 & 102 & 5 & 1 & 3.54 \\
Wezzle2D & 35 & 2.18 & 87 & 13 & 181 & 5 & 0 & 4.26 \\
ZKoss & 185 & 15.95 & 394 & 76 & 684 & 0 & 0 & 33.95 \\
Totals: & 2677 & 232.2 & 3321 & 652 & 6180 & 166 & 30 & 383.17 \\
\hline
\end{tabular}

TABLE I

EXPERIMENTAL RESULTS. JAVA8 IS THE JAVA. PACKAGE OF THE JDK 8.

to-one correspondence between source and target methods. In these cases, choosing any of the source methods passing preconditions to migrate would be safe as the nonmigrated methods would override the new default method, as such, we migrate the largest number of equivalent source methods, as seen with AbstractList.isEmpty() and AbsStack.isEmpty(). For this, we categorize viable source method bodies into equivalence sets, which are deemed equivalent by performing an AST differencing algorithm [17] after fully qualifying any constituent elements. Methods in the largest set are chosen for migration, while the others fail preconditions. Although exploring other techniques is a topic of future work, this case accounted for $0.31 \%$ of failures.

\section{Evaluation}

Implementation. Our approach is implemented as an open source plug-in to the Eclipse IDE, chosen for its existing refactoring framework [18] and that it is completely open source for all Java development. Eclipse ASTs with source symbol bindings were used as an intermediate representation. Our implementation is completely separate from the type constraints generated by the Eclipse Java Developer Tools.

To increase real-world applicability, we relaxed the closedworld assumption ( $\$ \mathrm{IV}$. For example, if an input method's destination interface is outside of the considered source code, it is conservatively labeled as non-migratable. Several options for reducing client impact exist, e.g., empty skeletal implementation classes can be either removed or deprecated. Furthermore, if such classes extend a super class not implementing all of the implemented interfaces, regardless of client code, the class is not removed. We additionally require no mismatches involving exception throws clauses and return types between source and target methods. An option to not consider non-standard (outside java.lang) annotation differences is available.

Experimental Evaluation. 19 open-source Java applications and libraries of varying size and domain (Table I) were used to 


\begin{tabular}{lll}
\hline \# & Precondition & Fails \\
\hline P1 & MethodContainsInconsistentParameterAnnotations & 1 \\
P2 & MethodContainsCallToProtectedObjectMethod & 1 \\
P3 & TypeVariableNotAvailable & 10 \\
P4 & DestinationInterfaceIsFunctional & 17 \\
P5 & TargetMethodHasMultipleSourceMethods & 19 \\
P6 & MethodContainsIncompatibleParameterTypeParameters & 42 \\
P7 & NoMethodsWithMultipleCandidateDestinations & 53 \\
P8 & TypeNotAccessible & 64 \\
P9 & SourceMethodImplementsMultipleMethods & 72 \\
P10 & SourceMethodProvidesImplementationsForMultipleMethods & 79 \\
P11 & MethodContainsTypeIncompatibleThisReference & 79 \\
P12 & IncompatibleMethodReturnTypes & 104 \\
P13 & ExceptionTypeMismatch & 105 \\
P14 & MethodContainsSuperReference & 147 \\
P15 & SourceMethodOverridesClassMethod & 258 \\
P16 & AnnotationMismatch & 305 \\
P17 & SourceMethodAccessesInstanceField & 463 \\
P18 & MethodNotAccessible & 1,679 \\
P19 & FieldNotAccessible & 2,565 \\
\hline
\end{tabular}

TABLE II

PRECONDITION FAILURES

evaluate our approach's effectiveness. Column KL is the number of non-blank, non-comment thousands of lines of code, ranging from $\sim 2 \mathrm{~K}$ for $r \mathrm{dp} 4 \mathrm{j}$ to $\sim 600 \mathrm{~K}$ for Azureus. The analysis was executed 5 times on an Intel Xeon E5 machine with 8 cores and 15GB RAM and a 8GB maximum heap size. Running time is shown in column tm (s), averaging $\sim 0.144$ secs/KLOC, which is practical even for large applications.

Default Method Migration: Column KM is the number of all methods in thousands. These are separated into two categories; the first is (filtered) methods that definitely cannot be refactored or be participating in the targeted design pattern. This includes methods not meeting the following criteria: (i) ones whose source is available, writable, and not generated, (ii) non-native, concrete instance methods (excluding constructors) declared in abstract classes implementing at least one interface whose source is also available, writable, and not generated, (iii) neither synchronized nor final, and (iv) overriding at least one non-default interface method. The remaining are candidates (column cnds). That these methods are declared in interface-implementing abstract classes is a strong indication that they are participating in the targeted pattern, i.e., they are meant to be subclassed and override an interface method. This increases the likelihood that these methods are general enough to be suitable default methods.

Our approach was able to automatically migrate $19.63 \%$, accounting for 652 methods across 19 projects, of the methods that could possibly be participating in the targeted skeletal implementation pattern (column dflts for defaults) in spite of its conservative nature. Since we migrate methods having only a single target, it is unlikely that a more suitable default implementation exists, with the only possible caveat being methods that are eliminated in smaller equivalence sets, but this accounted for only $0.31 \%$ of failure cases (explained next).

The tool was unable to refactor the remaining $80.37 \%$ of candidate methods. Column fps portrays the total number of failed preconditions, averaging $\sim 2.32$ per unmigratable method. Table $\Pi$ categorizes failures by kind, many of which correspond to the situations illustrated in \$III. others are more fine-grained. For instance, $\mathrm{F} 3$ and $\mathrm{F} 6$ occurs when there is either a method- or type-level mismatch in the number of type parameters or when they are not assignment compatible between source and target methods, respectively. F5 occurs for methods residing in non-selected equivalence sets, while P7 corresponds to the situation where there are ambiguous destination interfaces. $\mathrm{P} 9$ and $\mathrm{P} 10$ coincide with violations of type constraints 25] and 26, respectively, whereas $\mathrm{F} 11$ is a violated type constraint involving this in the source method body. $\mathrm{P} 8, \mathrm{P} 18$, and $\mathrm{F} 19$ arise when the source method body accesses (e.g., private) elements not visible from or inherited by the destination interface, and $\mathrm{F}[15$ preserves program semantics by circumventing tie breakers to classes. Failures of type P17 involve accessible fields violating constraint (7).

Skeletal Implementation Class Removal: Column $\delta$ depicts the number of skeletal implementation classes that contained methods that were migrated. Of those, column $-\delta$ shows the number that can be safely removed, constituting $18.07 \%$. The remaining $81.93 \%$ were not removable largely because the class was not completely empty after the refactoring, accounting for $66.02 \%, 18.93 \%$ had a super class not implementing a destination interface, and $15.05 \%$ had no super class but subclasses had incompatible super references. The total number of references to removable classes (not shown) averaged $\sim 9$ per removed class and was as high as 106 .

Discussion: Precondition failures are not unequivocally due to the analysis capabilities or conservative nature of our approach; portions are solely due to language constraints and/or limitations, and reporting these may give significant insight to language designers. $\mathrm{P} 2$, e.g., could be fixed in Java by having interfaces extend object. To alleviate errors arising from F10, designers could choose to not allow the squelching of default methods up the hierarchy by abstract methods down the hierarchy. In other words, the ability of subclasses to "hide" default methods could be removed; a similar consideration is discussed in [3, §3.6]. Insight is also given into how well this new language construct integrates into existing code, thus answering some of the questions raised by [3, §10].

Conversely, several failures may be alleviated by either further analysis or transformation. P8. $\mathrm{F} 19$, and $\mathrm{F} 18$, e.g., may sometimes be mitigated by prompting developers to increase element visibility. For P18, the inaccessible method could be declared in the destination interface if each implementer already provides an implementation. P[17 may sometimes be reduced by a composite refactoring that first encapsulates the field and then copies the setter/getter declarations to the destination interface. Note, however, this would not have fixed the field access failures related to size() and setsize() in Fig. 1(a) as they already encapsulate size.

While each of these are interesting areas of future work, they may require manual input from the developer or result in more invasive source code changes. In favor of a conservative, automated solution, we chose not to implement them at this time so that developers can take advantage of default methods on a mass scale with minimal code changes. P10 could be fixed 
by creating a delegate method in the source method's declaring type that calls the migrated default method. However, doing so does not reduce bloat, one of the motivations of this work.

As discussed previously, $18.07 \%$ of classes containing migrated methods could be completely removed. Thus, $81.93 \%$ of such classes were only partially migrated to interfaces. Despite the small percentage of completely migrated classes, there are many benefits to partial migration, including forgoing the need to extend a skeletal implementation class when the interface default methods suffice and less maintenance between the interface and skeletal class for the migrated methods (e.g., modifying duplicate method header annotations) [5].

Pull Request Study. To assess our approach's usability, we also submitted pull requests to popular open source Java projects on GitHub. We ensured that projects compiled correctly and had identical unit test results and compiler warnings before and after the refactoring. In some instances, minor manual intervention was required, e.g., to merge javadoc. Due to space limitations, study details can be found on our website http://cuny.is/interefact. Of the issued 19 pull requests, 4 have been successfully merged, 5 are open, and 10 were closed without merging. Ranging in size and domain, the merged requests are to projects and frameworks from organizations such as Eclipse, AOL, and NHL (National Hockey League), and include the popular Eclipse (formally Goldman Sachs) Collections framework. In all, the merged projects total 163 watches, 1071 stars, and 180 forks. Several other projects, although enthusiastic about the approach, rejected our pull requests, citing reasons such as that they had not yet moved to Java 8 or needed to support older Java clients at the time. We expect demand for Java 8 features to increase, especially since they are now (at least partially) supported by Android [19].

As the code's organization is changing, our refactoring may have engineering and design implications. While improving design is a motivating factor, enhancing modularity and reducing library bloat are others (see \$II). The acceptance of our refactoring results, totaling the addition of 349 and the removal of 647 LOC, to large and popular frameworks during this study increases confidence that the resulting design is acceptable.

Verifiability: Our plug-in is available at http://git.io/v2nX0 and subject source is available on our aforementioned website.

\section{RELATED WORK}

Goetz [10] also formalizes (using [20], an early version of) default methods. Constraints (25) and (26) have similar purposes as the type checking rules there, however, [10] does not deal with exceptions, functional interface invalidation, method body compatibility, and class removal. Type constraints are also well-established in many refactoring approaches.

A constraint-based approach to refactoring is also utilized in [21]. There, constraints are generated to assist refactorings in avoiding access control violations. [22] also develops constraints for averting such violations but for use in a broader transformation framework to simplify refactorings. These problems are related yet orthogonal to ours, however, these approaches could be used to augment our approach. [23] tackles the foresight problem typically found in constraint-based refactorings where new constraints are needed as a result of applying the refactoring. However, since our approach moves (method) bodies rather than declarations, no new constraints should be necessary as a result of the move.

Similar to the PUll UP METHOD refactoring [7], the "move original method to superclass" law [24] uses rules expressing when such a transformation is semantically equivalent. The declaration constraints there, however, do not apply here since no method declarations are being moved but rather bodies. Our approach fundamentally builds upon the other constraints, and we could have expanded this law to account for multiple inheritance issues and tie breakers with classes.

Our preliminary work [5] solely explored the feasibility of improving modularity via default methods. [25] inquires about using default methods for trait-oriented programming or mixins. [26] also analyzes differences between arguments and parameters names. Many refactorings use type constraints for type checking [7], type inference [27--29], and semantics preservation [30]. [7]3132] deal with reorganizing type hierarchies, [33] and [34] refactor Java programs to use lambda expressions and enumerated types, respectively. [35] demacrofies $\mathrm{C}++11$ programs. [36] refactors to use cloud-based services, and [37] automatically migrates CSS to preprocessors.

\section{CONCLUSION \& FutURE WORK}

We have presented an efficient, fully-automated, type constraint-based, semantics-preserving approach, featuring an exhaustive rule set, that migrates the skeletal implementation pattern in legacy Java code to instead use default methods. It is implemented as an Eclipse IDE plug-in and was evaluated on 19 open source projects. The results show that our tool scales and was able to refactor, despite its conservativeness and language constraints, $19.63 \%$ of all methods possibly participating in the pattern with minimal intervention. Our study highlights pattern usage and gives insight to language designers on applicability to existing software. Moreover, 4 pull requests were merged into GitHub repositories, which include large, widely used frameworks from reputable organizations.

In the future, we will explore pattern variations, e.g., allowing input methods from concrete classes, which requires analyzing instantiations and determining suitable default methods from concrete classes. Compensating for source methods directly accessing fields or methods outside destination interfaces will also be investigated, as well as javadoc merging, transforming build scripts for migrations across modules, other heuristics for method equivalence set elimination, machine learning to disambiguate destination interfaces, improving efficiency [38], and alternate testing techniques [39. 42].

\section{ACKNOWLEDGMENT}

Many thanks to Olivia Moore and Md Arefin for their assistance with experiments. This material is based upon work supported by PSC-CUNY under award \#69165-00 47 and the Tokyo Institute of Technology Research Abroad program. 


\section{REFERENCES}

[1] Oracle Corporation, "Java Programming Language Enhancements." [Online]. Available: http://docs.oracle.com/javase/8/docs/technotes/ guides/language/enhancements.html

[2] —-, "Default methods," 2016. [Online]. Available: http://docs.oracle.com/javase/tutorial/java/IandI/ defaultmethods.html

[3] B. Goetz, "Interface evolution via virtual extensions methods," Oracle Corporation, Tech. Rep., Jun. 2011. [Online]. Available: http://cr.openjdk.java.net/ briangoetz/lambda/Defender\%20Methods\%20v4.pdf

[4] J. Bloch, Effective Java. Prentice Hall, 2008.

[5] R. Khatchadourian, O. Moore, and H. Masuhara, "Towards improving interface modularity in legacy java software through automated refactoring," in Companion Proceedings of the $15^{\text {th }}$ International Conference on Modularity, ser. MODULARITY Companion 2016. New York, NY, USA: ACM, 2016, pp. 104-106.

[6] J. Palsberg and M. I. Schwartzbach, Object-oriented type systems. John Wiley and Sons Ltd., 1994.

[7] F. Tip, R. M. Fuhrer, A. Kieżun, M. D. Ernst, I. Balaban, and B. De Sutter, "Refactoring using type constraints," ACM Transactions on Programming Languages and Systems, vol. 33, no. 3, pp. 9:1-9:47, May 2011.

[8] M. Fowler, Refactoring: Improving the Design of Existing Code. Addison-Wesley Professional, 1999.

[9] Oracle Corporation, "Java ${ }^{\mathrm{TM}}$ platform, standard edition 8 api," 2016. [Online]. Available: http://docs.oracle.com/ javase/8/docs/api

[10] B. Goetz and R. Field, "Featherweight defenders: A formal model for virtual extension methods in java," Oracle Corporation, Tech. Rep., 2012. [Online]. Available: http://cr.openjdk.java.net/ briangoetz/lambda/ featherweight-defenders.pdf

[11] E. Gamma, R. Helm, R. Johnson, and J. Vlissides, Design patterns: elements of reusable object-oriented software. Boston, MA, USA: Addison-Wesley, 1995.

[12] C. S. Horstmann, Java SE 8 for the Really Impatient. Addison-Wesley Professional, 2014.

[13] J. Gosling, B. Joy, G. L. S. Jr., G. Bracha, and A. Buckley, The Java Language Specification, 8th ed. AddisonWesley Professional, 2014.

[14] G. B. Singh, "Single versus multiple inheritance in object oriented programming," SIGPLAN OOPS Mess., vol. 6, no. 1, pp. 30-39, Jan. 1995.

[15] M. Sakkinen, "Disciplined inheritance," in European Conference on Object-Oriented Programming, 1989.

[16] A. Lundblad, "Java: Do interfaces inherit from object?" 2016. [Online]. Available: http://programming.guide/ java/do-interfaces-inherit-from-object.html

[17] B. Fluri, M. Wuersch, M. PInzger, and H. Gall, "Change distilling: Tree differencing for fine-grained source code change extraction," IEEE Transactions on Software Engineering, vol. 33, no. 11, pp. 725-743, Nov. 2007.
[18] D. Bäumer, E. Gamma, and A. Kiezun, "Integrating refactoring support into a Java development tool," in ACM SIGPLAN International Conference on ObjectOriented Programming, Systems, Languages, and Applications, 2001.

[19] Google Inc., "Use java 8 language features I android developers." [Online]. Available: http: //developer.android.com/guide/platform/j8-jack.html

[20] A. Igarashi, B. C. Pierce, and P. Wadler, "Featherweight java: A minimal core calculus for java and gj," $A C M$ Transactions on Programming Languages and Systems, vol. 23, no. 3, pp. 396-450, May 2001.

[21] F. Steimann and A. Thies, "From public to private to absent: Refactoring java programs under constrained accessibility," in European Conference on Object-Oriented Programming, ser. ECOOP'09. Berlin, Heidelberg: Springer-Verlag, 2009, pp. 419-443.

[22] M. Schafer, A. Thies, F. Steimann, and F. Tip, "A comprehensive approach to naming and accessibility in refactoring java programs," IEEE Transactions on Software Engineering, vol. 38, no. 6, pp. 1233-1257, Nov. 2012.

[23] F. Steimann and J. von Pilgrim, "Constraint-based refactoring with foresight," in European Conference on Object-Oriented Programming, ser. ECOOP'12. Berlin, Heidelberg: Springer-Verlag, 2012, pp. 535-559.

[24] P. Borba, A. Sampaio, A. Cavalcanti, and M. Cornélio, "Algebraic reasoning for object-oriented programming," Science of Computer Programming, vol. 52, no. 1-3, pp. 53-100, Aug. 2004.

[25] V. Bono, E. Mensa, and M. Naddeo, "Trait-oriented programming in java 8," in Principles and Practices of Programming on the Java Platform: Virtual Machines, Languages, and Tools, ser. PPPJ '14. New York, NY, USA: ACM, 2014, pp. 181-186.

[26] H. Liu, Q. Liu, C.-A. Staicu, M. Pradel, and Y. Luo, "Nomen est omen: Exploring and exploiting similarities between argument and parameter names," in International Conference on Software Engineering, ser. ICSE '16. New York, NY, USA: ACM, 2016, pp. 1063-1073.

[27] A. Kiezun, M. D. Ernst, F. Tip, and R. M. Fuhrer, "Refactoring for parameterizing java classes," in International Conference on Software Engineering, ser. ICSE '07. Washington, DC, USA: IEEE Computer Society, 2007, pp. 437-446.

[28] R. Fuhrer, F. Tip, A. Kieżun, J. Dolby, and M. Keller, "Efficiently refactoring java applications to use generic libraries," in European Conference on Object-Oriented Programming, ser. ECOOP'05. Berlin, Heidelberg: Springer-Verlag, 2005, pp. 71-96.

[29] I. Balaban, F. Tip, and R. Fuhrer, "Refactoring support for class library migration," in ACM SIGPLAN International Conference on Object-Oriented Programming, Systems, Languages, and Applications, ser. OOPSLA '05. New York, NY, USA: ACM, 2005, pp. 265-279.

[30] B. De Sutter, F. Tip, and J. Dolby, "Customization of 
java library classes using type constraints and profile information," in European Conference on Object-Oriented Programming. Springer Berlin Heidelberg, 2004, pp. 584-608.

[31] I. Moore, "Automatic inheritance hierarchy restructuring and method refactoring," in ACM SIGPLAN International Conference on Object-Oriented Programming, Systems, Languages, and Applications, ser. OOPSLA '96. New York, NY, USA: ACM, 1996, pp. 235-250.

[32] Z. Alshara, A.-D. Seriai, C. Tibermacine, H. L. Bouziane, C. Dony, and A. Shatnawi, "Migrating large objectoriented applications into component-based ones: Instantiation and inheritance transformation," in ACM SIGPLAN International Conference on Generative Programming: Concepts and Experiences, ser. GPCE 2015. New York, NY, USA: ACM, 2015, pp. 55-64.

[33] A. Gyori, L. Franklin, D. Dig, and J. Lahoda, "Crossing the gap from imperative to functional programming through refactoring," in ACM SIGSOFT Symposium on the Foundations of Software Engineering, ser. ESEC/FSE 2013. New York, NY, USA: ACM, 2013, pp. 543-553.

[34] R. Khatchadourian, "Automated refactoring of legacy java software to enumerated types," Automated Software Engineering, pp. 1-31, 2016.

[35] A. Kumar, A. Sutton, and B. Stroustrup, "Rejuvenating $\mathrm{c}++$ programs through demacrofication," in International Conference on Software Maintenance, ser. ICSM '12. Washington, DC, USA: IEEE Computer Society, 2012, pp. 98-107.

[36] Y.-W. Kwon and E. Tilevich, "Cloud refactoring: Automated transitioning to cloud-based services," Automated Software Engineering, vol. 21, no. 3, pp. 345-372, Sep. 2014.

[37] D. Mazinanian and N. Tsantalis, "Migrating cascading style sheets to preprocessors by introducing mixins," in International Conference on Automated Software Engineering, ser. ASE 2016. New York, NY, USA: ACM, 2016, pp. 672-683.

[38] J. Kim, D. Batory, D. Dig, and M. Azanza, "Improving refactoring speed by 10x," in International Conference on Software Engineering, ser. ICSE '16. New York, NY, USA: ACM, 2016, pp. 1145-1156.

[39] X. Ge and E. Murphy-Hill, "Manual refactoring changes with automated refactoring validation," in International Conference on Software Engineering, ser. ICSE 2014. New York, NY, USA: ACM, 2014, pp. 1095-1105.

[40] G. Soares, R. Gheyi, and T. Massoni, "Automated behavioral testing of refactoring engines," IEEE Transactions on Software Engineering, vol. 39, no. 2, pp. 147-162, Feb. 2013.

[41] M. Mongiovi, "Safira: A tool for evaluating behavior preservation," in Proceedings of the ACM International Conference Companion on Object Oriented Programming Systems Languages and Applications Companion, ser. OOPSLA '11. New York, NY, USA: ACM, 2011, pp. 213-214.
[42] B. Daniel, D. Dig, K. Garcia, and D. Marinov, "Automated testing of refactoring engines," in ACM SIGSOFT Symposium on the Foundations of Software Engineering, ser. ESEC-FSE '07. New York, NY, USA: ACM, 2007, pp. 185-194. 